\title{
Discordant Alzheimer's neurodegenerative biomarkers and their clinical outcomes in older adults without dementia
}

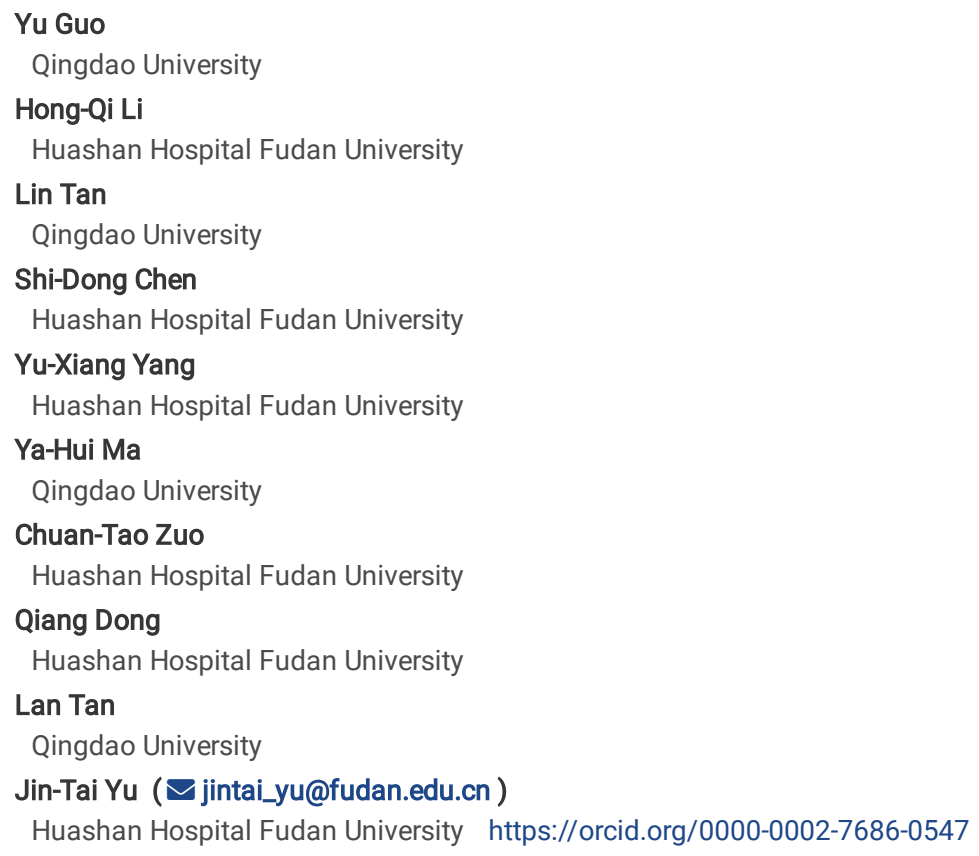




\begin{abstract}
Background In 2018 ATN framework, Alzheimer's neurodegenerative biomarkers comprised cerebrospinal fluid (CSF) total tau, 18 F-fluorodeoxyglucosepositron emission tomography, and brain atrophy. Although these biomarkers could be used interchangeably, some cases had discordant neurodegenerative biomarkers. Our aim was to assess the clinical outcomes of having discordant Alzheimer's neurodegenerative biomarkers.

Methods A total of 721 non-demented individuals from the Alzheimer's Disease Neuroimaging Initiative database were included and then further categorized into concordant negative, discordant, and concordant positive groups. Demographic distributions of the groups were compared. Longitudinal changes in clinical outcomes and risk of conversion were assessed using linear mixed-effects models and multivariate Cox proportional hazard models, respectively.

Results Discordant group was intermediate to concordant-negative and concordant-positive groups in terms of APOE $\varepsilon 4$ positivity, CSF amyloid-beta and phosphorylated tau. Compared with concordant-negative group, discordant group deteriorated faster in cognitive scores (Mini-Mental State Examination, the Clinical Dementia Rating Scale-Sum of Boxes, and the Functional Activities Questionnaire) and demonstrated greater rates of atrophy in brain structures (hippocampus, entorhinal cortex and whole brain), and concordant-positive group performed worse over time than discordant group. Moreover, the risk of cognitive progression increased from concordant-negative to discordant to concordant-positive. The longitudinal results were validated in $\mathrm{A}+\mathrm{T}+$, cognitively normal and mild cognitive impairment individuals, and were also validated by applying different cut-offs for neurodegenerative biomarkers.
\end{abstract}

Conclusions Discordant neurodegenerative status denotes a stage of cognitive function which is intermediate between concordant-negative and concordantpositive. Identification of discordant cases would provide insights into intervention and new therapy approaches, particularly in A+T+ individuals. Moreover, this work may be a complement to the ATN scheme.

\title{
Background
}

In 2018, the National Institute on Aging-Alzheimer's Association (NIA-AA) updated an "ATN" classification scheme used in Alzheimer's disease (AD) [1]. In this system, " $A$ " biomarkers reflect amyloidosis characterized by low cerebrospinal fluid (CSF) amyloid-beta (AB) or increased retention of $A \beta$ positron emission tomography (PET) tracers; "T" biomarkers refer to the elevated CSF phosphorylated tau ( $\mathrm{p}$-tau) or cortical tau PET; and "N" biomarkers are indicators of neurodegeneration or neuronal injury based on elevated CSF total tau (t-tau), hypometabolism on ${ }^{18} \mathrm{~F}$-fluorodeoxyglucose (FDG)-PET, or brain atrophy on magnetic resonance image (MRI) [1]. Individuals can be considered to be biologically diagnosed with AD when having both characteristic A $\beta$ and tau pathologies $(A+T+)$ [1]. These proposed biomarkers in the framework have been incorporated in research, which could facilitate and rationalize early diagnosis of diseases $[2,3]$.

Neurodegeneration, especially synapse loss, is the aspect of AD neuropathological changes that is most closely related to symptoms [4]. Therefore, the " $\mathrm{N}$ " biomarker panel provides vital pathological staging information [1] and exploring these biomarkers in actual clinical practice is necessary. Although the three "N" biomarkers can be used interchangeably, it is worth noting that heterogeneity exists among them [5]. CSF t-tau probably shows the intensity of neuronal damage at a specific time point [5-8]; brain atrophy on MRI reveals cumulative loss and shrinkage of the neuropil [9-11]; and FDG-PET may reflect atrophy of the neuropil and impaired function of neurons. All these differences among " $\mathrm{N}$ " biomarkers likely lead to discordance [12-16]. Discordant biomarkers potentially have significant implications for neurobiological mechanisms of biomarker discrepancies and AD neuropathogenesis [17]. Prior studies mainly focused on the associations of neurodegenerative biomarkers or only a single biomarker rather than investigation into the combination of multiple neurodegenerative markers utilizing various modalities [18-22]. However, whether and how " $\mathrm{N}$ " biomarker discordance affects clinical outcomes is currently understudied. Herein, we conducted the first longitudinal study to compare discordant group with concordant-negative and concordant-positive groups in terms of baseline demographic distributions and longitudinal clinical outcomes. We hypothesized that the discordant status might denote different stages of disease pathogenesis.

\section{Methods}

\section{Alzheimer's Disease Neuroimaging Initiative (ADNI) Study Design}

Data used in the preparation of this article were obtained from the ADNI database (http://adni.loni.usc.edu) [23, 24]. The ADNI was launched in 2003 as a public-private partnership with the primary goal of testing the effectiveness of integrating neuroimaging, clinical, biological, and neuropsychological markers in measuring the progression of mild cognitive impairment $(\mathrm{MCl})$ and early AD. All ADNI participants have been recruited from more than 50 sites across the United States and Canada.

\section{Standard protocol approvals, registrations, and patient consents}

Regional ethical committees of all institutions approved the ADNI study. Written informed consent was obtained from all participants or authorized representatives

\section{Participants}

Subjects from the ADNI prospective clinical cohort were included in this article if they received baseline and periodic clinical, neuropsychological and CSF assessments as well as serial MRI and FDG-PET examinations. To avoid the impact of AD on results, we included only non-demented subjects diagnosed as cognitively normal $(\mathrm{CN})$ controls and $\mathrm{MCl}$ patients. For detailed diagnostic criteria, see .

\section{CSF Measurements}


CSF samples were collected and shipped on dry ice to the ADNI Biomarker core laboratory at the University of Pennsylvania Medical Center. Aliquots ( $0.5 \mathrm{~mL}$ ) were prepared from these samples and stored in barcode-labeled polypropylene vials at $-80^{\circ} \mathrm{C}$. The CSF proteins, including CSF A, $\mathrm{p}$-tau and t-tau, were measured using the multiplex xMAP Luminex platform (Luminex Corp, Austin, TX) with the INNOBIA AlzBio3 kit (Innogenetics) [25].

\section{Neuroimaging and Cognition}

Structural MRI brain scans were acquired by Siemens Trio 3.0 T or Vision 1.5 T scanners. Automated volume estimates were processed with Free-surfer software version 4.3 and 5.1 for the 1.5T and 3.0 T MRI images, respectively. Regions of interests (ROIs) included hippocampus, entorhinal cortex and whole brain.

FDG-PET data were from UC Berkeley and Lawrence Berkeley National Laboratory on the website (http://adni.loni.ucla.edu/). The PET images were spatially normalized in SPM to the standard template. For FDG-PET, we averaged counts of angular, temporal, and posterior cingulate regions.

General cognition was assessed by Mini-Mental State Examination (MMSE), Clinical Dementia Rating Scale-Sum of Boxes (CDRSB), and Functional Activities Questionnaire (FAQ) scores.

\section{Grouping of subjects}

Group classifications were determined by normal (-) and abnormal (+) biomarker results at baseline. Based on the cutoff thresholds of biomarkers reported in previous articles, $A \beta$-positive $(A+)$ subjects were those who had CSF A $\beta$ levels $\leq 192 \mathrm{pg} / \mathrm{ml}$ [26]. P-tau-positive ( $T+$ ) was defined as a value above the cutoff point of $23 \mathrm{pg} / \mathrm{ml}$ [26]. CSF t-tau-positive $(\mathrm{N}+$ ) referred to the levels $\geq 93 \mathrm{pg} / \mathrm{ml}$ [26]. The estimated total intracranial volume (eTIV) was applied to adjust the hippocampal volume (HPV) with the following equation: adjusted hippocampal volume (aHPV) = Raw HPV - b (eTIV - Mean eTIV), where b indicated the regression coefficient when HPV was regressed against eTIV [27]. The aHPV-positive ( $\mathrm{N}+$ ) was defined as the cutoff point $\leq 6723$ mm ${ }^{3}$ [27]. We defined FDGPET-positive $(\mathrm{N}+)$ and negative $(\mathrm{N}-)$ subjects based on a cutoff point of 1.21 [26].

To validate the main results derived from the above reported cutoffs, we applied different cutoff values. Firstly, we excluded borderline values within $\pm 5 \%$ of the above cut-off value, to avoid drawing conclusions based on borderline cases that easily could be misclassified due to variable measurements. The cut-offs used in the article were thus: CSF t-tau $+\geq 97.65 \mathrm{pg} / \mathrm{ml}$, aHPV $+\leq 6386.85 \mathrm{~mm}^{3}$ and FDG-PET $+\leq 1.1495$. Secondly, a biomarker classification was done via the receiver operating characteristics (ROC) analyses (see "Statistical analyses" section), which produced the new cut-off values: CSF t-tau $+\geq 68.65 \mathrm{pg} / \mathrm{ml}$, $\mathrm{aHPV}+\leq 6586.14 \mathrm{~mm}^{3}$ and FDG-PET $+\leq 1.1776$.

The subjects were then categorized into three profiles depending on the status of neurodegenerative biomarkers: CSF/PET profile (CSF-/PET- group, CSF-/PET + group, CSF+/PET- group, and CSF+/PET + group), CSF/MRI profile (CSF-/MRI- group, CSF-/MRI + group, CSF+/MRI- group, and CSF+/MRI+ group), and $\mathrm{PET} / \mathrm{MRI}$ profile (PET-/MRI- group, PET-/MRI + group, $\mathrm{PET}+/ \mathrm{MRI}$ - group, and PET+/MRI + group).

\section{Statistical analyses}

We tested group differences using the Kruskal-Wallis analyses for continuous variables and chi-square tests for categorical data. Continuous variables were presented as means (standard deviations (SDs)) and categorical variables as numbers (percents). Cognitive scores and brain volumes were $z$ log-transformed to normalize the distributions, and their longitudinal changes were performed in linear mixed-effects models. In the analyses for cognitive scores, we adjusted for baseline age, gender, APOE $\varepsilon 4$, and years of education. In the analyses for brain volumes, baseline age, gender, APOE $\varepsilon 4$, and total intracranial volume (TIV) were included as covariates. To access the risk of cognitive progression ( $C N$ converted to $\mathrm{MCl}$ or $\mathrm{AD}$; $\mathrm{MCl}$ converted to $\mathrm{AD}$ at follow-up), we constructed unadjusted Kaplan-Meier (KM) plots. Then we ran multivariate Cox proportional hazard models adjusted for baseline age, gender, educational years, and APOE $\varepsilon 4$ status. Besides, we conducted subgroup analyses by clinical diagnosis $(\mathrm{CN} / \mathrm{MCl})$. To explore the influence of pathologic changes in $A D$, we constricted the population to $A+T+$ and replicated the above longitudinal analyses. Furthermore, ROC analyses were conducted to determine the thresholds of neurodegenerative biomarkers that most efficiently distinguished $C N$ from $A D$ individuals (the data used here included $A D$ population). The maximum value of the Youden's index (sensitivity + specificity -1 ) was defined as the new cutoff point. Statistical significance was defined as $P<0.05$ (two-sided). Statistical analyses were completed using R software (version 3.5.1) and IBM SPSS Statistics 23.

\section{Results}

\section{Baseline Characteristics}

This study included 721 subjects $(\mathrm{CN}=279, \mathrm{MCl}=442)$ without removing borderline cases (Table 1$)$. The mean (SD) age of the participants was $72.5(6.8)$ years, $46.5 \%$ were women, and $98.1 \%$ had more than 12 years of education. By applying the previously proposed cut-offs, the classification of subjects resulted in three profiles (Fig. 1): CSF/PET profile (394 CSF-/PET-, 126 CSF-/PET+, 112 CSF+/PET-, 89 CSF+/PET+), CSF/MRI profile (387 CSF-/MRI-, 133 CSF-/MRI+, 114 CSF+/MRI-, 87 CSF+/MRI+), and PET/MRI profile (392 PET-/MRI-, 114 PET-/MRI+, 109 PET+/MRI-, $106 \mathrm{PET+/MRI+).} \mathrm{Distribution} \mathrm{of} \mathrm{clinical}$ diagnosis (CN/MCl), age, APOE $\varepsilon 4$ positivity, MMSE, CDRSB, FAQ, aHPV, entorhinal volume, whole brain volume, FDG-PET, CSF A $\beta$, p-tau and t-tau were significantly different among concordant and discordant groups in the three profiles (appendix 1 ). Demographic information of $A+T+$ patients were summarized in appendix 2 . 
Table 1

Baseline Demographic Characteristics

\begin{tabular}{|c|c|c|c|c|c|c|c|c|c|}
\hline \multirow[t]{2}{*}{ Characteristics } & \multicolumn{3}{|c|}{ CSF/PET profile } & \multicolumn{3}{|c|}{ CSF/MRI profile } & \multicolumn{3}{|c|}{ PET/MRI profile } \\
\hline & CSF-/PET- & Discordant & CSF+/PET+ & CSF-/MRI- & Discordant & CSF+/MRI+ & PET-/MRI- & Discordant & $\mathrm{PET}+/ \mathrm{MF}$ \\
\hline $\mathrm{N}$ & 394 & 238 & 89 & 387 & 247 & 87 & 392 & 223 & 106 \\
\hline $\begin{array}{l}\text { Baseline } \\
\mathrm{CN} / \mathrm{MCl}\end{array}$ & $187 / 207$ & $77 / 161$ & $15 / 74$ & $199 / 188$ & $68 / 179$ & $12 / 75$ & 200/192 & $67 / 156$ & $12 / 94$ \\
\hline Age (years) & $71.3(6.5)$ & $74.0(6.6)$ & $73.8(7.1)$ & 70.8 (6.3) & $73.9(6.9)$ & $76.3(6.0)$ & $70.8(6.5)$ & $74.2(6.7)$ & $75.2(6.2)$ \\
\hline Female & $189(48 \%)$ & $106(45 \%)$ & $40(45 \%)$ & $180(47 \%)$ & $110(45 \%)$ & $45(52 \%)$ & $203(52 \%)$ & $86(39 \%)$ & $46(43 \%)$ \\
\hline $\begin{array}{l}\text { Years of } \\
\text { education }\end{array}$ & $16.4(2.6)$ & $15.9(2.7)$ & $16.2(2.9)$ & $16.4(2.5)$ & $16.0(2.9)$ & $16.1(2.8)$ & $16.3(2.6)$ & $16.3(2.7)$ & $15.9(3.0)$ \\
\hline $\begin{array}{l}\text { APOE } \varepsilon 4 \\
\text { positive }\end{array}$ & 115 (29\%) & $116(49 \%)$ & $63(71 \%)$ & $120(31 \%)$ & $115(47 \%)$ & $59(68 \%)$ & $132(34 \%)$ & $93(42 \%)$ & $69(65 \%)$ \\
\hline MMSE & $28.7(1.4)$ & $28.1(1.7)$ & $27.4(1.9)$ & $28.8(1.3)$ & $28.1(1.7)$ & $27.3(1.9)$ & $28.7(1.4)$ & $28.2(1.6)$ & $27.2(1.9)$ \\
\hline CDRSB & $0.6(0.8)$ & $1.1(1.0)$ & $1.6(1.1)$ & $0.6(0.8)$ & $1.1(1.0)$ & $1.6(1.1)$ & $0.6(0.7)$ & $1.1(1.0)$ & $1.6(1.1)$ \\
\hline FAQ & $1.0(2.4)$ & $2.5(4.0)$ & $3.9(3.8)$ & $1.0(2.4)$ & $2.6(3.8)$ & $3.9(4.2)$ & $0.8(2.0)$ & $2.7(3.9)$ & $4.1(4.3)$ \\
\hline aHPV $\left(\mathrm{mm}^{3}\right)$ & $\begin{array}{l}7,500.6 \\
(919.9)\end{array}$ & $\begin{array}{l}6,839.5 \\
(1,038.9)\end{array}$ & $\begin{array}{l}6,541.1 \\
(955.2)\end{array}$ & $\begin{array}{l}7,766.6 \\
(638.9)\end{array}$ & $\begin{array}{l}6,680.6 \\
(985.3)\end{array}$ & $\begin{array}{l}5,855.7 \\
(595.7)\end{array}$ & $\begin{array}{l}7,781.4 \\
(648.9)\end{array}$ & $\begin{array}{l}6,717.4 \\
(877.8)\end{array}$ & $\begin{array}{l}5,819.8 \\
(709.8)\end{array}$ \\
\hline $\begin{array}{l}\text { Entorhinal } \\
\text { volume }\left(\mathrm{mm}^{3}\right)\end{array}$ & $\begin{array}{l}3,886.1 \\
(619.2)\end{array}$ & $\begin{array}{l}3,585.1 \\
(732.5)\end{array}$ & $\begin{array}{l}3,376.5 \\
(652.7)\end{array}$ & $\begin{array}{l}3,917.8 \\
(561.8)\end{array}$ & $\begin{array}{l}3,521.4 \\
(712.5)\end{array}$ & $\begin{array}{l}3,151.5 \\
(648.0)\end{array}$ & $\begin{array}{l}3,911.5 \\
(583.6)\end{array}$ & $\begin{array}{l}3,562.4 \\
(642.0)\end{array}$ & $\begin{array}{l}3,124.3 \\
(721.6)\end{array}$ \\
\hline $\begin{array}{l}\text { Whole brain } \\
\text { volume }\left(\mathrm{mm}^{3}\right)\end{array}$ & $\begin{array}{l}1,066,857.7 \\
(100,242.8)\end{array}$ & $\begin{array}{l}1,037,127.3 \\
(108,716.9)\end{array}$ & $\begin{array}{l}1,026,394.9 \\
(93,711.3)\end{array}$ & $\begin{array}{l}1,070,554.1 \\
(99,248.9)\end{array}$ & $\begin{array}{l}1,035,742.3 \\
(103,813.4)\end{array}$ & $\begin{array}{l}1,005,309.8 \\
(99,323.9)\end{array}$ & $\begin{array}{l}1,067,953.9 \\
(100,028.7)\end{array}$ & $\begin{array}{l}1,039,788.2 \\
(97,865.7)\end{array}$ & $\begin{array}{l}1,010,17 € \\
(112,807 .\end{array}$ \\
\hline FDG-PET & $1.3(0.1)$ & $1.2(0.1)$ & $1.1(0.1)$ & $1.3(0.1)$ & $1.2(0.1)$ & $1.2(0.1)$ & $1.4(0.1)$ & $1.2(0.1)$ & $1.1(0.1)$ \\
\hline $\begin{array}{l}\text { CSF A } \beta \\
(\mathrm{pg} / \mathrm{mL})\end{array}$ & $\begin{array}{l}201.9 \\
(48.0)\end{array}$ & $\begin{array}{l}165.7 \\
(51.1)\end{array}$ & $\begin{array}{l}141.7 \\
(36.9)\end{array}$ & $\begin{array}{l}203.0 \\
(47.9)\end{array}$ & $\begin{array}{l}164.2 \\
(49.1)\end{array}$ & $\begin{array}{l}143.3 \\
(42.0)\end{array}$ & $\begin{array}{l}196.8 \\
(50.5)\end{array}$ & $\begin{array}{l}175.6 \\
(51.4)\end{array}$ & $\begin{array}{l}144.3 \\
(41.0)\end{array}$ \\
\hline $\begin{array}{l}\text { CSF p-tau } \\
\text { (pg/mL) }\end{array}$ & $29.6(14.7)$ & $41.8(22.8)$ & $58.9(23.0)$ & $29.0(14.4)$ & $45.2(25.6)$ & $51.6(16.7)$ & $34.7(20.1)$ & $37.6(22.3)$ & 45.8 (21.c \\
\hline $\begin{array}{l}\text { CSF t-tau } \\
(\mathrm{pg} / \mathrm{mL})\end{array}$ & $56.2(17.7)$ & $94.2(47.8)$ & $\begin{array}{l}144.5 \\
(43.1)\end{array}$ & $55.1(17.8)$ & $95.0(45.1)$ & $\begin{array}{l}145.4 \\
(45.7)\end{array}$ & $69.5(37.6)$ & $85.4(46.2)$ & $\begin{array}{l}104.9 \\
(55.8)\end{array}$ \\
\hline
\end{tabular}

Discordant group in the CSF/PET, CSF/MRI, and PET/MRI profiles represent combined group of CSF-/PET + and CSF+/PET-, combined group of CSF-/MRI + and $\mathrm{CSF}+/ \mathrm{MRI}$-, and combined group of PET-/MRI + and PET+/MRI-, respectively.

Abbreviations: AB, amyloid beta; aHPV, adjusted hippocampal volume; CDRSB, Clinical Dementia Rating Scale-Sum of Boxes; CN, cognitively normal; CSF, cerebrospinal fluid; FAQ, Functional Activities Questionnaire; FDG, ${ }^{18}$ F-fluorodeoxyglucose; $\mathrm{MCl}$, mild cognitive impairment; MMSE, Mini-Mental State Examination; MRI, Magnetic Resonance Imaging; PET, positron emission tomography; p-tau, phosphorylated tau; t-tau, total tau.

The distribution plots of APOE $\varepsilon 4, C S F A \beta$, and p-tau were shown in Fig. 2. There was an increase in the proportion of APOE $\varepsilon 4$ positivity from concordantnegative to discordant to concordant-positive in the three profiles. The APOE $\varepsilon 4$ allele frequency differed between CSF-/MRI + and CSF+/MRI- groups (39\% versus $55 \%, \mathrm{P}=0.011$ ), whereas it was difficult to distinguish between CSF-/PET + and CSF+/PET- groups or between PET-/MRI + and PET+/MRI- groups. The trend was similar in CSF p-tau in the three profiles, as discordant patients had more CSF p-tau accumulations than concordant-negative individuals and concordant-positive subjects had more CSF p-tau deposits than discordant patients. There were significant differences in CSF p-tau burden between $\mathrm{CSF}+/ \mathrm{PET}$ - and CSF-/PET + groups as well as between CSF+/MRI- and CSF-/MRI + groups (both $\mathrm{P}<0.001$ ). Additionally, the concentration of CSF A 3 reduced from concordant-negative to discordant to concordant-positive in the three profiles. CSF+/PET- and CSF+/MRI- patients showed lower CSF A 3 levels than CSF-/PET + and CSF-/MRI + patients, respectively (both $\mathrm{P}=0.006$ ).

\section{Longitudinal Changes in cognitive scores and brain structures}

In the longitudinal analyses of cognitive scores (Fig. 3), we adjusted for age, gender, APOE $\varepsilon 4$, and years of education at baseline. In terms of MMSE score over time, discordant group performed better than concordant-positive group $(\mathrm{P}<0.0001)$, and demonstrated an accelerated decline than concordant-negative group $(\mathrm{P}<0.0001)$. MMSE scores declined faster in CSF-/PET + patients than CSF+/PET- patients $(\mathrm{P}=0.0406)$, and there were no differences in MMSE scores between CSF-/MRI + and CSF+/MRI- patients, as well as between PET-/MRI + and PET+/MRI- patients. There was an upward trend in the rates of increase in CDRSB and FAQ scores from concordant-negative to discordant to concordant-positive in the three profiles. CSF+/PET- patients had a faster accrual of FAQ scores than CSF-/PET + patients $(\mathrm{P}=0.0020)$ (appendix 3 and 4). In subgroup analyses, $\mathrm{MCl}$ patients who had more abnormal "N" biomarkers performed worse over time on various cognitive assessments (MMSE, CDRSB, and FAQ) in the three profiles. With respect to CN subjects, this tendency was only significant in CDRSB scores (appendix 5). 
In the longitudinal analyses of brain structures (Fig. 4), we adjusted for age, gender, APOE $\varepsilon 4$, and TIV at baseline. There was a growing tendency for the rates of deterioration of hippocampal, entorhinal and whole brain structures from concordant-negative to discordant to concordant-positive in the three profiles. $\mathrm{CSF}+$ /PET- patients had faster rates of hippocampal atrophy than CSF-/PET + patients $(P=0.0474)$. However, no group differences were detected in entorhinal or whole brain atrophy rates among discordant subjects (appendix 3 and 4). In subgroup analyses, $\mathrm{MCl}$ patients who had more abnormal " $\mathrm{N}$ " biomarkers displayed accelerated reduction in brain volumes in the three profiles. As for CN subjects, hippocampal atrophy rates were greater in the CSF+/PET + group than the combined group of CSF-/PET + and CSF+/PET-, and entorhinal atrophy rates were greater in the combined group of CSF-/MRI + and CSF+/MRI- than the CSF-/MRI- group (appendix 5).

In A + T + patients, cognitive performance in cognitive scores (MMSE, CDRSB and FAQ) worsened from concordant-negative to discordant to concordantnegative. In terms of brain structures (hippocampus, entorhinal cortex and whole brain), the combined group of CSF-/MRI + and CSF+/MRI- as well as the $\mathrm{PET}+/ \mathrm{MRI}+$ group displayed higher atrophy rates than the CSF-/MRI- group as well as the combined group of PET-/MRI + and PET+/MRI-, respectively.

Moreover, hippocampus and whole brain atrophied faster in the combined group of CSF-/PET + and CSF+/PET-, compared with the CSF-/PET- group (appendix 6 and 7).

\section{Analyses of cognitive progression}

In Cox regression models, we adjusted for age, gender, educational level, and APOE $\varepsilon 4$ status. The progression risk in the CSF+/PET + group was $2.6147-$ times higher than that of the combined group of CSF-/PET + and CSF+/PET-, and the latter group had a 3.3275-times risk higher than the CSF-/PET- group. Discordant patients within CSF/MRI profile had a greater conversion rate than CSF-/MRI- individuals (HR $=4.0255,95 \% \mathrm{Cl}=2.8093-5.7680)$, and CSF+/MRI + patients progressed faster compared with discordant individuals ( $\mathrm{HR}=2.0081,95 \% \mathrm{Cl}=1.4338-2.8130)$. Similarly, discordant patients in $\mathrm{PET} / \mathrm{MRI}$ profile were more likely to progress than those in the PET-/MRI- group with the HR of $3.9705(95 \% \mathrm{Cl}=2.7735-5.6840)$, and PET+/MRI + participants displayed an increased risk of conversion than discordant participants with the HR of $1.9915(95 \% \mathrm{Cl}=1.4366-2.7607)$ (Fig. 5A, appendix 8). Besides, we did not detect any intergroup differences in the risk of conversion among discordant patients in the three profiles (appendix 9 and 10). Then we performed subgroup analyses stratified by clinical diagnosis. In $\mathrm{MCl}$ patients, the cognitive progression rate increased from concordant-negative to discordant to concordant-positive in the three profiles. Nonetheless, among CN subjects, no significant difference was observed in the risk of conversion between discordant and concordant-positive groups in both CSF/PET and PET/MRI profiles. And no significant intergroup differences were detected among discordant subjects in both $\mathrm{CN}$ and $\mathrm{MCl}$ individuals (appendix 11 and 12).

In the $\mathrm{A}+\mathrm{T}+$ patients, the $\mathrm{CSF}+\mathrm{PET}+$ group had a greater risk of progression than discordant group with the $\mathrm{HR}$ of $2.3200(95 \% \mathrm{Cl}=1.6382-3.2856)$, and the discordant group had a 3.1766-times risk higher than the CSF-/PET- group. Similarly, in CSF/MRI and PET/MRI profiles, discordant subjects progressed faster than concordant-negative subjects, and concordant-positive subjects progressed faster than discordant patients. CSF-/PET + subjects showed a 2.1276-fold risk of cognitive progression compared with CSF+/PET- participants, and CSF-/MRI + individuals displayed a 1.6591-fold risk than that of CSF+/MRIparticipants (Fig. 5B, appendix 13 and 14).

\section{Validation of the main results using other cut-offs for neurodegenerative biomarkers}

Different group classifications were also made to ensure that the main results were not caused by random classifications:

(1) When applying previously proposed cut-offs by excluding borderline cases (CSF t-tau $+\geq 97.65 \mathrm{pg} / \mathrm{ml}$, aHPV $+\leq 6386.85 \mathrm{~mm}^{3}$ and FDG-PET $+\leq 1.1495$ ), 380 individuals were included (appendix 15 and 16). Patients with more abnormal neurodegenerative biomarkers had a greater risk of cognitive deterioration in the three profiles (appendix 17 and 18). Besides, CSF-/PET + subjects were more likely to progress than CSF+/PET-individuals.

(2) We also used new cut-offs derived from the ROC analyses (CSF t-tau $+\geq 68.65 \mathrm{pg} / \mathrm{ml}$, aHPV $+\leq 6586.14 \mathrm{~mm}^{3}$ and FDG-PET $\left.+\leq 1.1776\right)($ appendix 19 and 20). The more abnormal neurodegenerative biomarkers the patients had, the higher progression risk they displayed (appendix 21 and 22 ). Besides, CSF-/PET + and PET+/MRI- participants showed higher progression rates in comparison with CSF+/PET- and PET-/MRI + individuals, respectively.

\section{Discussion}

In the present study, we found that discordant group was intermediate to concordant-negative and concordant-positive groups in terms of APOE $\varepsilon 4$ positivity, CSF $A \beta$ and $p$-tau levels at baseline, as well as the rates of cognitive decline reflected by cognitive scores and brain structures. Besides, the risk of cognitive progression increased from concordant-negative to discordant to concordant-positive. These longitudinal results were validated in $\mathrm{A}+\mathrm{T}+\mathrm{CN}$ and $\mathrm{MCl}$ individuals, and were also validated by applying different cut-offs for neurodegenerative biomarkers. Altogether, our findings suggest that discordant neurodegenerative status denotes a stage of cognitive function which is intermediate between concordant-negative and concordant-positive.

Neurodegenerative pathology can be reliably measured in vivo with neuroimaging technology or CSF assessments, but substantial discordance exists when utilizing different methods to evaluate the " $\mathrm{N}$ " biomarkers in the same person [1]. Several surrogate mechanisms were possibly helpful in explaining the discordant states. Firstly, discordant cases accounted for a large proportion in the whole study sample, and consequently differences might exist in the time point at which neurodegenerative changes were detected by CSF assessments, MRI or FDG-PET. Vemuri et al. reported that MRI could be closer correlated with cognitive development than CSF t-tau, since the latter might be more prone to diurnal physiologic variations, thus revealing transient rather than cumulative damage [21]. However, this finding was inconsistent with our longitudinal results from discordant subjects. Thus, it's difficult to determine the sequence of "N" biomarker abnormality and find which specific " $\mathrm{N}$ " biomarker might indicate an earlier pathologic stage. Further research on this topic is needed. Secondly, the presence of the "N" biomarkers can be caused by several diseases, and therefore these biomarkers are not specific for neurodegenerative changes in AD. Also, in any individual, the proportion of neurodegenerative damage due to AD versus other probable comorbidities (most of which have no extant biomarker) remains unclear [1]. Therefore, the "N" biomarker positivity measured by CSF assessments or neuroimaging could be caused by other situations rather than 
$A D$, such as cerebrovascular disease (white matter hyperintensity) and neuroinflammation [28-30]. The third explanation is the existence of analytical artifacts. CSF may be absorbed on the surface of the tube, and its decreased amount that's available for analysis probably affected the obtained concentration in CSF [31]. However, neuroimaging technology is likely to bring false positive results in patients under comorbid conditions, and bring false negative results in patients with atypical forms of neurodegenerative pathology.

Since AD pathology has accumulated for decades before apparent clinical symptoms occur, the early identification of non-demented individuals at imminent risk of cognitive impairment would provide insights into intervention as well as new therapy approaches [32]. And the heterogeneity in the definition of neuronal injury is vital to clinical trials using biomarkers for enrollment or as alternative endpoint measures. In the three profiles, the discordant group was intermediate to concordant-negative and concordant-positive groups in terms of cognitive performance, no matter in non-demented, or $\mathrm{CN}$ or $\mathrm{MCl}$ populations. Accordingly, concordant-negative, discordant and concordant-positive groups were likely to indicate meaningfully different stages of cognitive function. Regardless of any abnormality in neuroimaging signatures (patterns of gray matter atrophy on structural MRI or FDG-PET) or CSF t-tau measurements, the isolated " $\mathrm{N}$ " positivity indicated a relatively early stage that needed to implement interventions before any two biomarkers became abnormal. Previous studies have reported the correlations between neuronal injury factors, and it has been noted that the combination of these biomarkers might provide better prediction than either source of data alone $[15,19,21,22]$. Vos et al. also suggested that individuals with both CSF A $\beta$ deposition and neuronal injury showed an increased risk of disease progression [16]. Nevertheless, due to the lack of evidence on whether and how discordant status influenced clinical outcomes, the feasibility and practicality of identifying discordant cases with cognitive disorders requires more research to confirm.

Our findings may have important implications for the diagnosis and treatment of $A D$, since they highlight the role of a discordant status in determining the therapeutic window before irreversible neuropathological changes. In the ATN system, "A" and "T" biomarkers reveal characteristic pathological changes that define $A D$, whereas neurodegenerative/neuronal injury biomarkers are nonspecific, which are applied only for staging of disease severity [1]. Targeting $A+T+$ patients, a recent study has compared the clinical outcomes of individuals having normal or abnormal single " $\mathrm{N}$ " biomarker and has found that all of the three "N" biomarkers were highly related to an increased risk of conversion to AD dementia [33]. By combining these "N" biomarkers, our study revealed that the conversion risk in discordant group was intermediate between those of concordant-negative and concordant-positive groups. In another word, even among the patients who have developed into a stage which could be biologically defined as AD, early identification of the patients with abnormal "N" biomarkers may provide insights into disease-modifying therapeutics or interventions of modifiable risk factors, which thus might delay the occurrence of cognitive decline or disease progression. In addition, our work could be a complement to the original ATN framework for AD's biological definition.

We conducted a large prospective study with a relatively long follow-up duration, which well characterized the cognitive trajectories of discordant and concordant patients. An additional strength was that results were robust even after threshold modification, or in different populations. Nonetheless, some caveats should be emphasized. Firstly, dichotomizing each biomarker may mask a potential continuum. Compared with the condition employing only one single biomarker, the classification error rate increased when three distinct " $N$ " biomarkers were utilized. Secondly, although the total sample size was large, the numbers of individuals in various groups were insufficient, especially when the discordant population was further categorized into different groups or when groups were stratified by clinical diagnosis, which may reduce the statistical power to detect longitudinal changes. Thirdly, the results targeting discordant subjects were inconsistent and it's difficult to conclude which " $\mathrm{N}$ " biomarker became abnormal first. Thus, further investigations targeting this topic were necessary.

\section{Conclusions}

In conclusion, this study supported that discordant neurodegenerative status denoted a stage of cognitive function intermediate between concordant-negative and concordant-positive. Identification of discordant cases would provide insights into intervention and disease-modifying therapeutics, particularly in $\mathrm{A}+\mathrm{T}+$ individuals. And this work could be a complement to the ATN framework. Moreover, further studies are warranted to determine the sequence of "N" biomarker abnormalities.

\section{Abbreviations}

$A \beta=$ amyloid-beta; $A D=$ Alzheimer's disease; $A D N I$ = Alzheimer's Disease Neuroimaging Initiative; aHPV = adjusted hippocampal volume; $C D R S B=C$ linical Dementia Rating Scale-Sum of Boxes; $C N$ = cognitively normal; $C S F=$ cerebrospinal fluid; eTIV = estimated total intracranial volume; $F A Q=F u n c t i o n a l$ Activities Questionnaire; FDG-PET = ${ }^{18} \mathrm{~F}$-fluorodeoxyglucose-positron emission tomography; $\mathrm{HPV}=$ hippocampal volume; $\mathrm{KM}=\mathrm{Kaplan}-\mathrm{Meier}$; $\mathrm{MCl}=$ mild cognitive impairment; MMSE = Mini-Mental State Examination; MRI = magnetic resonance image; NIA-AA = National Institute on Aging-Alzheimer's Association; $\mathrm{PET}=$ positron emission tomography; $\mathrm{p}$-tau = phosphorylated tau; ROC = receiver operating characteristics; ROIs = Regions of interests; $\mathrm{SDs}=$ standard deviations; TIV = total intracranial volume; $\mathrm{t}$-tau = total tau.

\section{Declarations}

\section{Ethics approval and consent to participate}

Regional ethical committees of all institutions approved the ADNI study. Written informed consent was obtained from all participants or authorized representatives.

\section{Consent for publication}

Not applicable. 


\section{Availability of data and materials}

The data used and analyzed in this study can be obtained from the ADNI database (http://adni.loni.usc.edu).

\section{Competing interests}

The authors declared no potential conflicts of interest with respect to the research, authorship, and/or publication of this article.

\section{Funding}

This study was supported by grants from the National Key R\&D Program of China (2018YFC1314700), the National Natural Science Foundation of China (91849126), Shanghai Municipal Science and Technology Major Project (No.2018SHZDZX01) and ZHANGJIANG LAB, Tianqiao and Chrissy Chen Institute, and the State Key Laboratory of Neurobiology and Frontiers Center for Brain Science of Ministry of Education, Fudan University. Data collection and sharing for this project was funded by the Alzheimer's Disease Neuroimaging Initiative (ADNI) (National Institutes of Health Grant U01 AG024904) and DOD ADNI (Department of Defense award number W81XWH-12-2-0012). ADNI is funded by the National Institute on Aging, the National Institute of Biomedical Imaging and Bioengineering, and through generous contributions from the following: AbbVie, Alzheimer's Association; Alzheimer's Drug Discovery Foundation; Araclon Biotech; BioClinica, Inc.; Biogen; Bristol-Myers Squibb Company; CereSpir, Inc.; Cogstate; Eisai Inc.; Elan Pharmaceuticals, Inc.; Eli Lilly and Company; Eurolmmun; F. Hoffmann-La Roche Ltd and its affiliated company Genentech, Inc.; Fujirebio; GE Healthcare; IXICO Ltd.; Janssen Alzheimer Immunotherapy Research \& Development, LLC.; Johnson \& Johnson Pharmaceutical Research \& Development LLC.; Lumosity; Lundbeck; Merck \& Co., Inc.; Meso Scale Diagnostics, LLC.; NeuroRx Research; Neurotrack Technologies; Novartis Pharmaceuticals Corporation; Pfizer Inc.; Piramal Imaging; Servier; Takeda Pharmaceutical Company; and Transition Therapeutics. The Canadian Institutes of Health Research is providing funds to support ADNI clinical sites in Canada. Private sector contributions are facilitated by the Foundation for the National Institutes of Health (www.fnih.org). The grantee organization is the Northern California Institute for Research and Education, and the study is coordinated by the Alzheimer's Therapeutic Research Institute at the University of Southern California. ADNI data are disseminated by the Laboratory for Neuro Imaging at the University of Southern California.

\section{Authors' contributions}

JTY conceptualized and designed the study. YG, HQL, and LT did the manuscript preparation and data acquisition. YG, HQL, LT, and JTY analyzed the data, performed statistical analysis and interpreted the data. YG, HQL, LT, SDC, YXY, YHM, and JTY wrote the first draft of the manuscript. All authors reviewed the manuscript. All authors have contributed to the manuscript revising and editing critically for important intellectual content and given final approval of the version and agreed to be accountable for all aspects of the work presented here. All authors read and approved the final manuscript.

\section{Acknowledgements}

The authors thank all the colleagues who have made contributions to build the ADNI database. The authors also thank the subjects and their family for their cooperation in this study.

\section{References}

1.

Jack CR Jr, Bennett DA, Blennow K, Carrillo MC, Dunn B, Haeberlein SB, Holtzman DM, Jagust W, Jessen F, Karlawish J, et al. NIA-AA Research Framework: Toward a biological definition of Alzheimer's disease. Alzheimers Dement. 2018;14:535-62.

2.

McKhann GM, Knopman DS, Chertkow H, Hyman BT, Jack CR Jr, Kawas CH, Klunk WE, Koroshetz WJ, Manly JJ, Mayeux R, et al. The diagnosis of dementia due to Alzheimer's disease: recommendations from the National Institute on Aging-Alzheimer's Association workgroups on diagnostic guidelines for Alzheimer's disease. Alzheimers Dement. 2011;7:263-9.

3.

Jack CR Jr, Albert MS, Knopman DS, McKhann GM, Sperling RA, Carrillo MC, Thies B, Phelps CH. Introduction to the recommendations from the National Institute on Aging-Alzheimer's Association workgroups on diagnostic guidelines for Alzheimer's disease. Alzheimers Dement. $2011 ; 7: 257-62$.

4.

Terry RD, Masliah E, Salmon DP, Butters N, DeTeresa R, Hill R, Hansen LA, Katzman R. Physical basis of cognitive alterations in Alzheimer's disease: synapse loss is the major correlate of cognitive impairment. Ann Neurol. 1991;30:572-80.

5 .

Blennow K, Hampel H. CSF markers for incipient Alzheimer's disease. Lancet Neurol. 2003;2:605-13.

6.

Buchhave P, Minthon L, Zetterberg H, Wallin AK, Blennow K, Hansson O. Cerebrospinal fluid levels of beta-amyloid 1-42, but not of tau, are fully changed already 5 to 10 years before the onset of Alzheimer dementia. Arch Gen Psychiatry. 2012;69:98-106.

7.

van Rossum IA, Vos SJ, Burns L, Knol DL, Scheltens P, Soininen H, Wahlund LO, Hampel H, Tsolaki M, Minthon L, et al. Injury markers predict time to dementia in subjects with $\mathrm{MCl}$ and amyloid pathology. Neurology. 2012;79:1809-16.

Page $7 / 13$ 
8.

Roe CM, Fagan AM, Grant EA, Hassenstab J, Moulder KL, Maue Dreyfus D, Sutphen CL, Benzinger TL, Mintun MA, Holtzman DM, Morris JC. Amyloid imaging and CSF biomarkers in predicting cognitive impairment up to 7.5 years later. Neurology. 2013;80:1784-91.

9.

Bobinski M, de Leon MJ, Wegiel J, Desanti S, Convit A, Saint Louis LA, Rusinek H, Wisniewski HM. The histological validation of post mortem magnetic resonance imaging-determined hippocampal volume in Alzheimer's disease. Neuroscience. 2000;95:721-5.

10.

Zarow C, Vinters HV, Ellis WG, Weiner MW, Mungas D, White L, Chui HC. Correlates of hippocampal neuron number in Alzheimer's disease and ischemic vascular dementia. Ann Neurol. 2005;57:896-903.

11.

Barkhof F, Polvikoski TM, van Straaten EC, Kalaria RN, Sulkava R, Aronen HJ, Niinisto L, Rastas S, Oinas M, Scheltens P, Erkinjuntti T. The significance of medial temporal lobe atrophy: a postmortem MRI study in the very old. Neurology. 2007;69:1521-7.

12.

Chetelat G, Ossenkoppele R, Villemagne VL, Perrotin A, Landeau B, Mezenge F, Jagust WJ, Dore V, Miller BL, Egret S, et al. Atrophy, hypometabolism and clinical trajectories in patients with amyloid-negative Alzheimer's disease. Brain. 2016;139:2528-39.

13.

Alexopoulos P, Kriett L, Haller B, Klupp E, Gray K, Grimmer T, Laskaris N, Forster S, Perneczky R, Kurz A, et al. Limited agreement between biomarkers of neuronal injury at different stages of Alzheimer's disease. Alzheimers Dement. 2014;10:684-9.

14.

Gordon BA, Friedrichsen K, Brier M, Blazey T, Su Y, Christensen J, Aldea P, McConathy J, Holtzman DM, Cairns NJ, et al. The relationship between cerebrospinal fluid markers of Alzheimer pathology and positron emission tomography tau imaging. Brain. 2016;139:2249-60.

15.

Toledo JB, Weiner MW, Wolk DA, Da X, Chen K, Arnold SE, Jagust W, Jack C, Reiman EM, Davatzikos C, et al. Neuronal injury biomarkers and prognosis in ADNI subjects with normal cognition. Acta neuropathologica communications. 2014;2:26.

16.

Vos SJB, Gordon BA, Su Y, Visser PJ, Holtzman DM, Morris JC, Fagan AM, Benzinger TLS. NIA-AA staging of preclinical Alzheimer disease: discordance and concordance of CSF and imaging biomarkers. Neurobiol Aging. 2016;44:1-8.

17.

de Wilde A, Reimand J, Teunissen CE, Zwan M, Windhorst AD, Boellaard R, van der Flier WM, Scheltens P, van Berckel BNM, Bouwman F, Ossenkoppele R. Discordant amyloid-beta PET and CSF biomarkers and its clinical consequences. Alzheimers Res Ther. 2019;11:78.

18.

Rubi S, Noguera A, Tarongi S, Oporto M, Garcia A, Vico H, Espino A, Picado MJ, Mas A, Pena C, Amer G. Concordance between brain (18)F-FDG PET and cerebrospinal fluid biomarkers in diagnosing Alzheimer's disease. Revista espanola de medicina nuclear e imagen molecular. 2018;37:3-8.

19.

Chiaravalloti A, Barbagallo G, Ricci M, Martorana A, Ursini F, Sannino P, Karalis G, Schillaci O. Brain metabolic correlates of CSF Tau protein in a large cohort of Alzheimer's disease patients: A CSF and FDG PET study. Brain research. 2018;1678:116-22.

20.

Caminiti SP, Ballarini T, Sala A, Cerami C, Presotto L, Santangelo R, Fallanca F, Vanoli EG, Gianolli L, lannaccone S, et al. FDG-PET and CSF biomarker accuracy in prediction of conversion to different dementias in a large multicentre $\mathrm{MCl}$ cohort. Neurolmage Clinical. 2018;18:167-77.

21.

Vemuri P, Wiste HJ, Weigand SD, Shaw LM, Trojanowski JQ, Weiner MW, Knopman DS, Petersen RC, Jack CR. Jr.. Alzheimer's Disease Neuroimaging I: MRI and CSF biomarkers in normal, $\mathrm{MCl}$, and AD subjects: diagnostic discrimination and cognitive correlations. Neurology. 2009;73:287-93.

22.

Ottoy J, Niemantsverdriet E, Verhaeghe J, De Roeck E, Struyfs H, Somers C, Wyffels L, Ceyssens S, Van Mossevelde S, Van den Bossche T, et al. Association of short-term cognitive decline and MCl-to-AD dementia conversion with CSF, MRI, amyloid-and (18)F-FDG-PET imaging. Neurolmage Clinical. $2019 ; 22: 101771$. 23.

Petersen RC, Aisen PS, Beckett LA, Donohue MC, Gamst AC, Harvey DJ, Jack CR Jr, Jagust WJ, Shaw LM, Toga AW, et al. Alzheimer's Disease Neuroimaging Initiative (ADNI): clinical characterization. Neurology. 2010;74:201-9.

24.

Saykin AJ, Shen L, Foroud TM, Potkin SG, Swaminathan S, Kim S, Risacher SL, Nho K, Huentelman MJ, Craig DW, et al. Alzheimer's Disease Neuroimaging Initiative biomarkers as quantitative phenotypes: Genetics core aims, progress, and plans. Alzheimers Dement. 2010;6:265-73.

25.

Shaw LM, Vanderstichele H, Knapik-Czajka M, Clark CM, Aisen PS, Petersen RC, Blennow K, Soares H, Simon A, Lewczuk P, et al. Cerebrospinal fluid biomarker signature in Alzheimer's disease neuroimaging initiative subjects. Ann Neurol. 2009;65:403-13.

26.

Jagust WJ, Landau SM, Shaw LM, Trojanowski JQ, Koeppe RA, Reiman EM, Foster NL, Petersen RC, Weiner MW, Price JC, et al. Relationships between biomarkers in aging and dementia. Neurology. 2009;73:1193-9.

27.

Mormino EC, Betensky RA, Hedden T, Schultz AP, Amariglio RE, Rentz DM, Johnson KA, Sperling RA. Synergistic effect of beta-amyloid and neurodegeneration on cognitive decline in clinically normal individuals. JAMA Neurol. 2014;71:1379-85.

Page $8 / 13$ 
Bos I, Verhey FR, Ramakers I, Jacobs HIL, Soininen H, Freund-Levi Y, Hampel H, Tsolaki M, Wallin AK, van Buchem MA, et al. Cerebrovascular and amyloid pathology in predementia stages: the relationship with neurodegeneration and cognitive decline. Alzheimers Res Ther. 2017;9:101.

29.

Soldan A, Pettigrew C, Zhu Y, Wang MC, Moghekar A, Gottesman RF, Singh B, Martinez O, Fletcher E, DeCarli C, et al. White matter hyperintensities and CSF Alzheimer disease biomarkers in preclinical Alzheimer disease. Neurology. 2019.

30 .

Niranjan R. Recent advances in the mechanisms of neuroinflammation and their roles in neurodegeneration. Neurochem Int. 2018;120:13-20.

31.

Hansson O, Mikulskis A, Fagan AM, Teunissen C, Zetterberg H, Vanderstichele H, Molinuevo JL, Shaw LM, Vandijck M, Verbeek MM, et al. The impact of preanalytical variables on measuring cerebrospinal fluid biomarkers for Alzheimer's disease diagnosis: A review. Alzheimers Dement. 2018;14:1313-33. 32.

Meyer PF, Binette AP, Gonneaud J, Breitner JCS, Villeneuve S. Characterization of Alzheimer Disease Biomarker Discrepancies Using Cerebrospinal Fluid Phosphorylated Tau and AV1451 Positron Emission Tomography. JAMA Neurol. 2020.

33.

Ou YN, Xu W, Li JQ, Guo Y, Cui M, Chen KL, Huang YY, Dong Q, Tan L, Yu JT. Alzheimer's Disease Neuroimaging I: FDG-PET as an independent biomarker for Alzheimer's biological diagnosis: a longitudinal study. Alzheimers Res Ther. 2019;11:57.

\section{Figures}

A

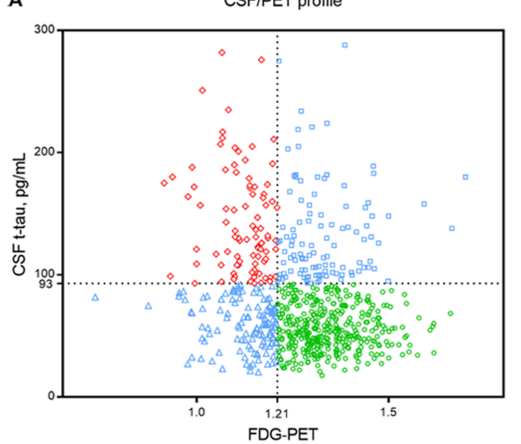

- CSF-/PET- $\triangle$ CSF-/PET+ O CSF+/PET- O CSF+/PET+
B

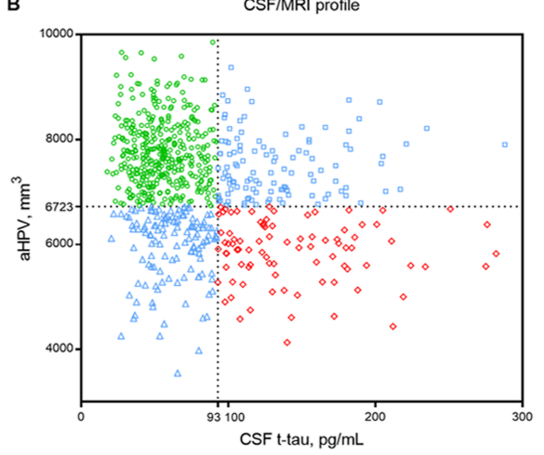

- CSF-/MRI- $\triangle \mathrm{CSF}-/ \mathrm{MRI}+\mathrm{CSF}+/ \mathrm{MRI}-\circ \mathrm{CSF}+\mathrm{MRI}+$
C

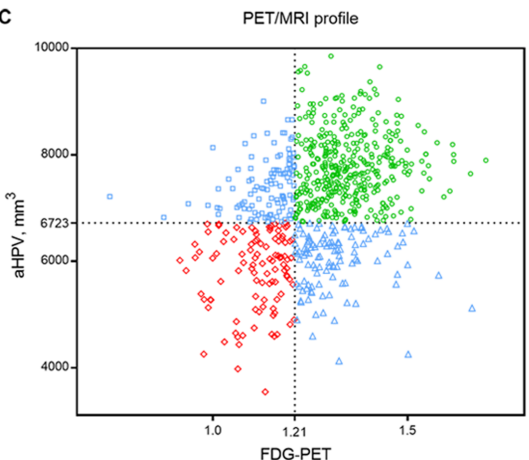

PET-/MRI-

PET-/MRI+ 。 PET+/MRI- 。 PET+/MRI+

Figure 1

Distribution plots of neurodegenerative biomarkers. (A) CSF/PET profile: CSF t-tau versus FDG-PET; (B) CSF/MRI profile: CSF t-tau versus aHPV; (C) PET/MRI profile: FDG-PET versus aHPV. Dashed lines represent the cutoff values for CSF t-tau, FDG-PET, and aHPV. Abbreviations: aHPV, adjusted hippocampal volume; CSF, cerebrospinal fluid; FDG, 18F-fluorodeoxyglucose; MRI, Magnetic Resonance Imaging; PET, positron emission tomography; t-tau, total tau. 
A CSFIPET profile

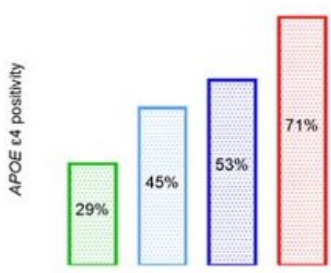

$\square$ CSF-IPET. $\square$ CSF-IPET+ 口 CSF+IPET. $\square$ CSF+PET +
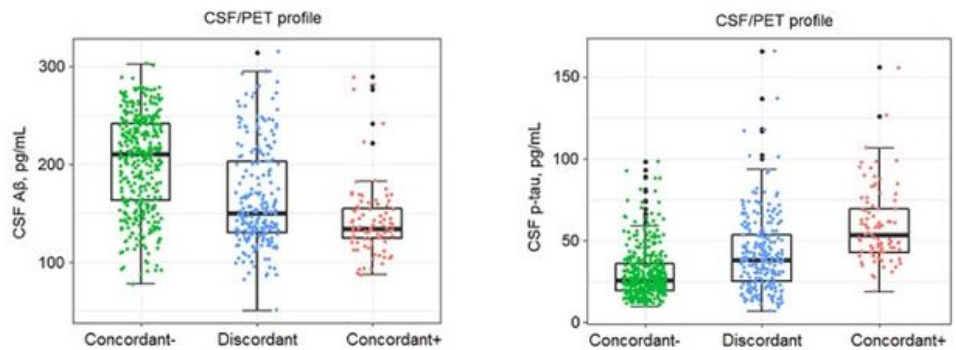

B

CSFMRI profile

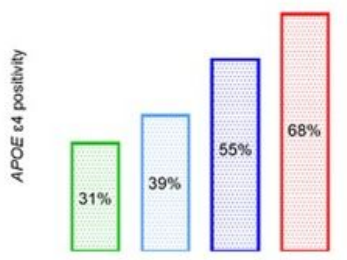

$\square$ CSF-MRI$\square \mathrm{CSF} \cdot / \mathrm{MRI}+$ $\square \mathrm{CSF}+$ /MRI$\square \mathrm{CSF}+/ \mathrm{MRI}+$
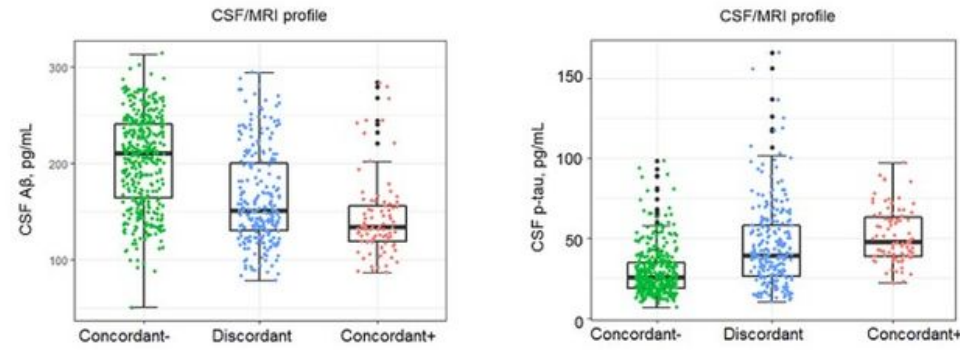

C PETMRI profile

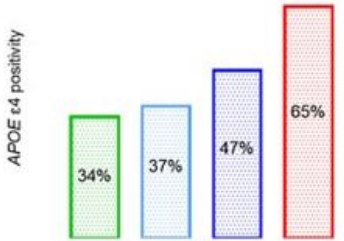

$\square$ PET-/MRI$\square$ PET-MRI+ $\square$ PET+/MRI+
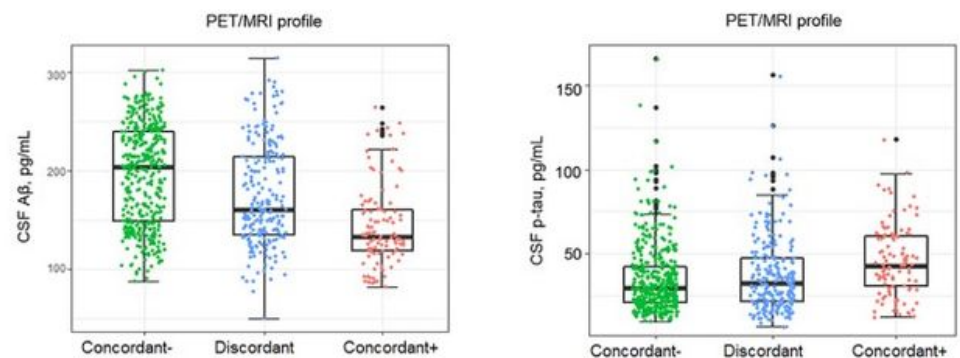

\section{Figure 2}

Differences in APOE $\varepsilon 4$ positivity, CSF A $\beta$, and p-tau levels. (A) CSF/PET profile; (B) CSF/MRI profile; (C) PET/MRI profile. Abbreviations: A 3 , amyloid beta; CSF, cerebrospinal fluid; MRI, Magnetic Resonance Imaging; PET, positron emission tomography; p-tau, phosphorylated tau. 

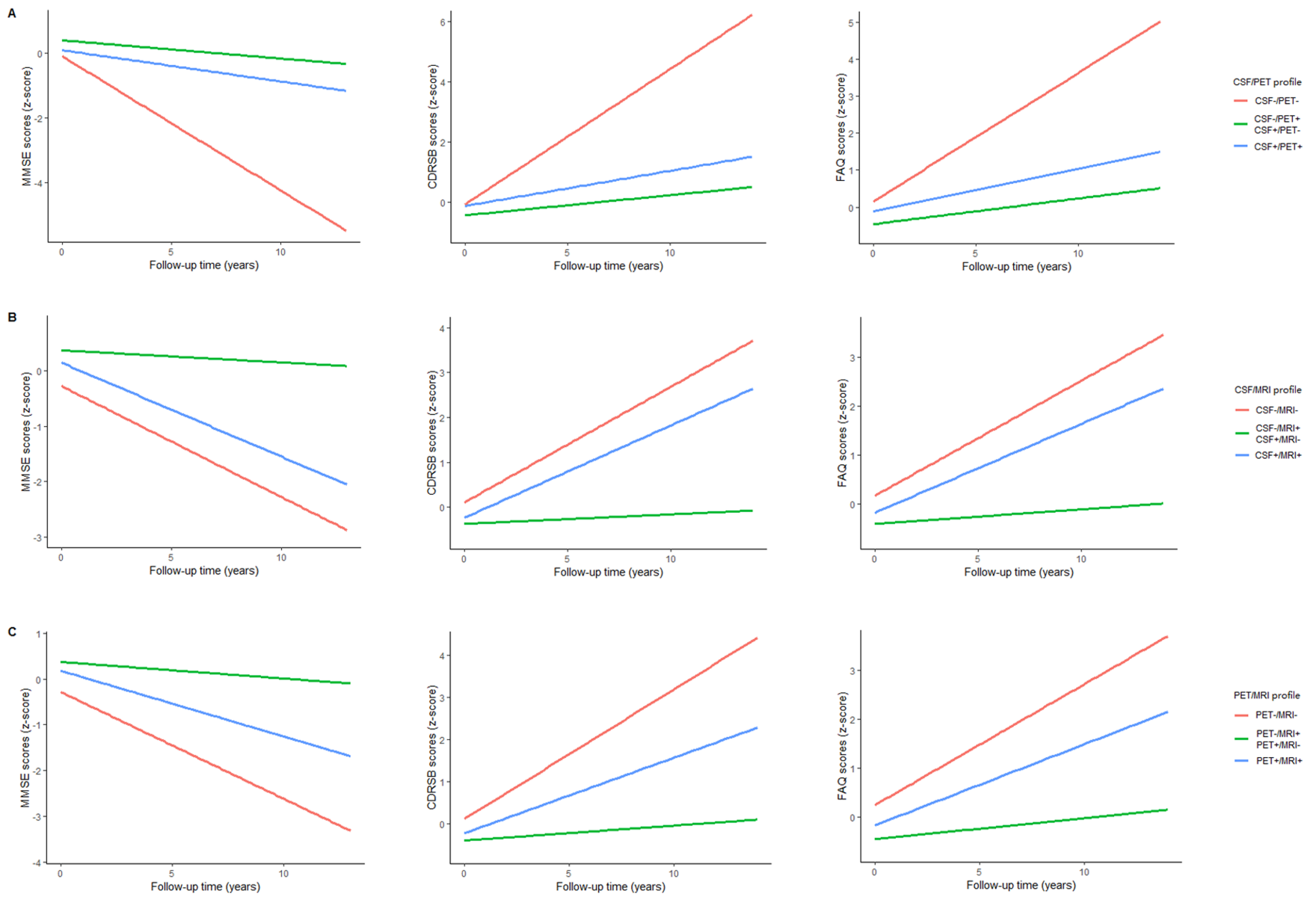

Figure 3

Longitudinal changes of cognitive scores in different profiles. (A) CSF/PET profile; (B) CSF/MRI profile; (C) PET/MRI profile. Abbreviations: CDRSB, Clinical Dementia Rating Scale-Sum of Boxes; CSF, cerebrospinal fluid; FAQ, Functional Activities Questionnaire; MMSE, Mini-Mental State Examination; MRI, Magnetic Resonance Imaging; PET, positron emission tomography. 

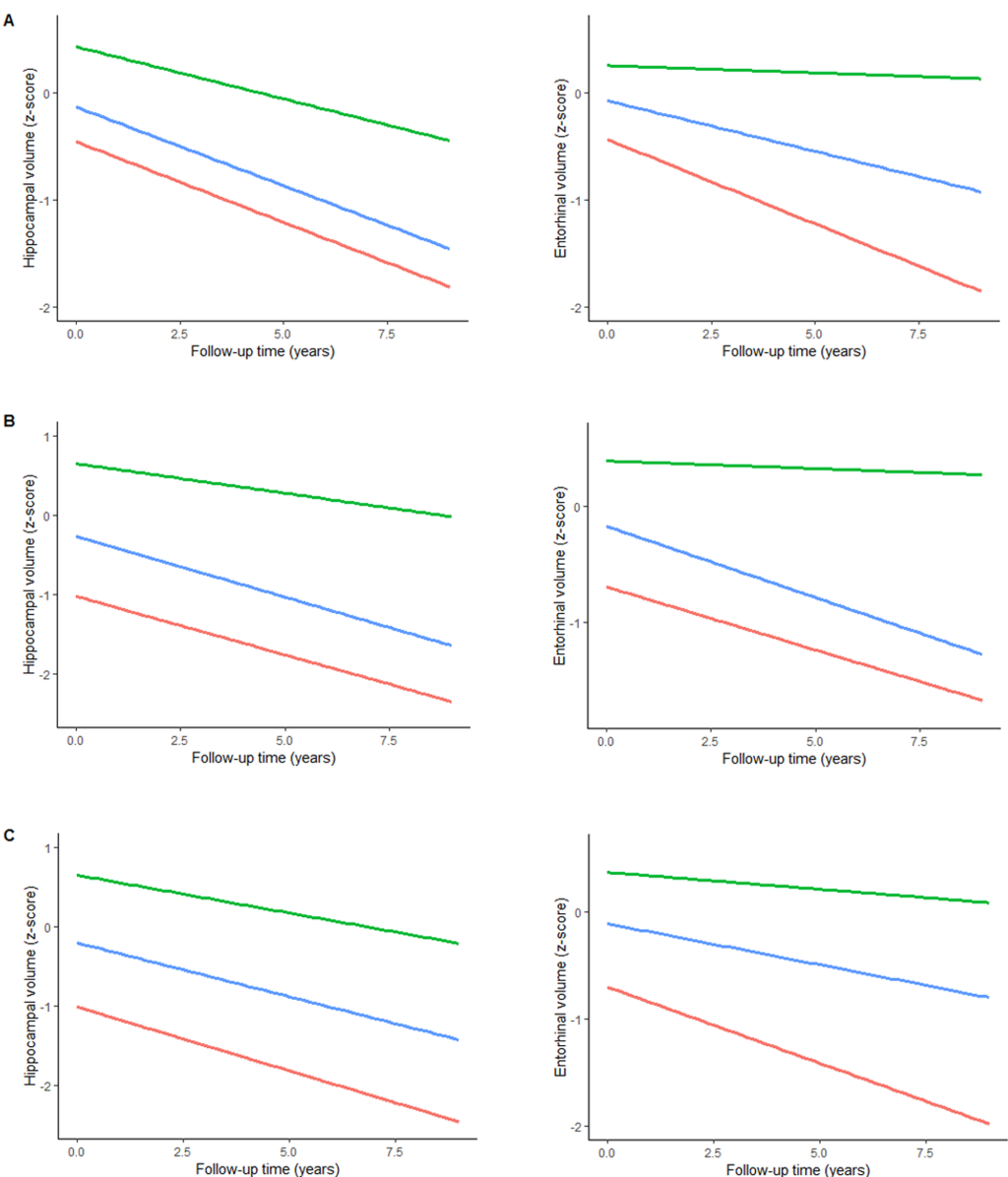

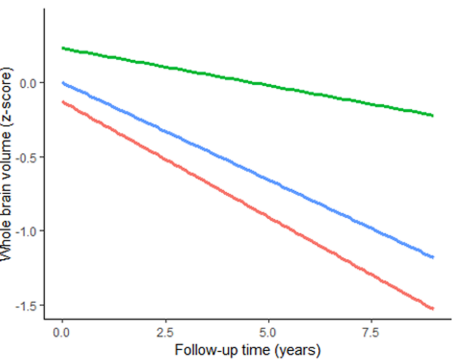

CSFIPET profile - CSF-IPET- CSF-IPET+ - CSF+PET+

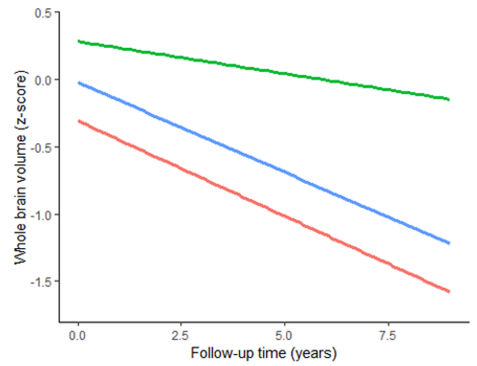

CSFMRI profile - CSF-MRI$-{ }_{\text {CSF }}^{\text {CSFMRI- }}$ - CSF+MRIt

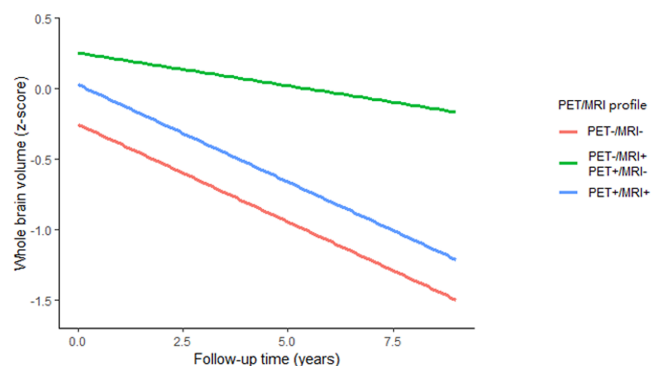

Figure 4

Longitudinal changes of brain structures in different profiles. (A) CSF/PET profile; (B) CSF/MRI profile; (C) PET/MRI profile. Abbreviations: CSF, cerebrospinal fluid; MRI, Magnetic Resonance Imaging; PET, positron emission tomography. 

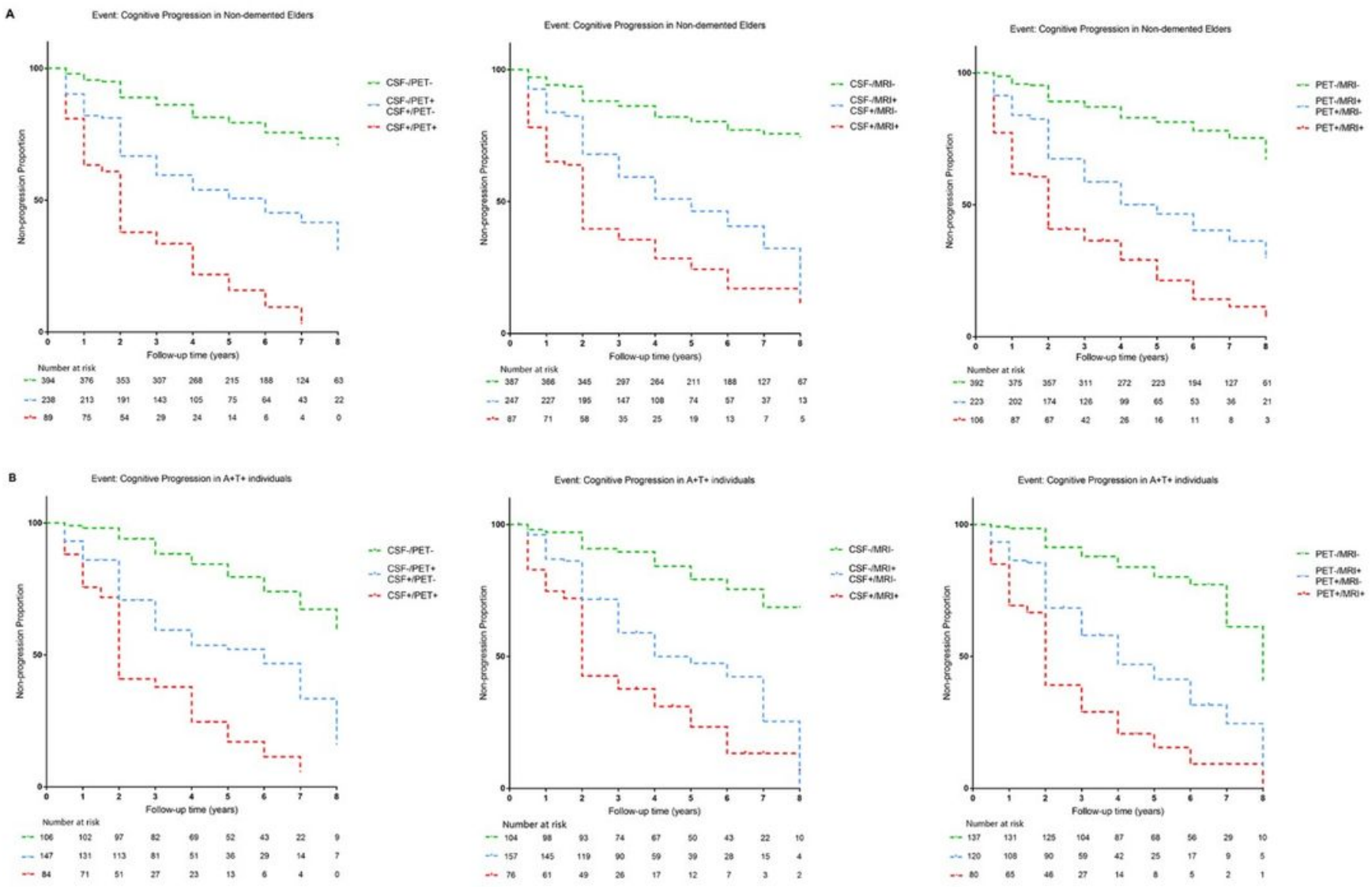

Figure 5

Survival analyses for probability of cognitive progression in (A) non-demented elders, and (B) A+T+ individuals. Comparisons of cognitive progression between concordant and discordant individuals were shown in CSF/PET, CSF/MRI and PET/MRI profiles. Numbers of individuals at risk at different follow-up time points were presented. Survival time was calculated according to the intervals from the baseline evaluation to the time points of cognitive progression. Abbreviations: CSF, cerebrospinal fluid; MRI, Magnetic Resonance Imaging; PET, positron emission tomography.

\section{Supplementary Files}

This is a list of supplementary files associated with this preprint. Click to download.

- Appendix.docx 